\title{
Diabetes care for emerging adults: transition from pediatric to adult diabetes care systems
}

\author{
Young Ah Lee, MD \\ Division of Endocrinology and \\ Metabolism, Department of \\ Pediatrics, Seoul National University \\ Children's Hospital, Seoul, Korea
}

With the increasing prevalence of diabetes mellitus in children, transitioning patients from childhood to adulthood are increasing. High-risk behaviors and poor glycemic control during the transition period increase the risk for hypoglycemia and hyperglycemia as well as chronic microvascular and macrovascular complications. Discussions regarding complications and preparations for transition must take place before the actual transition to adult care systems. Pediatric care providers should focus on diabetes self-management skills and prepare at least 1 year prior to the transfer. Pediatric providers should also provide a written summary about previous and current glycemic control, complications and the presence of mental health problems such as disordered eating behaviors and affective disorders. Transition care should be individualized, with an emphasis on diabetes self-management to prevent acute and long-term complications. Regular screening and management of complications should proceed according to pediatric and adult guidelines. Birth control, use of alcohol, smoking and driving should also be discussed. Barriers to self-management and care must be recognized and solutions sought. The goals of transitional care are to effectively transition the diabetic patient from the pediatric to adult care system with less elapsed time in between and to improve post-transition outcome. Previous studies regarding diabetes transitional care programs including patient education programs, medical coordinators and auxiliary service systems reported promising results. However, there is a lack of evidence regarding best practices in transition care. Further studies are needed to provide evidence based transitional care programs that take both medical and psychosocial aspects of diabetes care into consideration.

Keywords: Transition to adult care, Diabetes mellitus
Received: 9 September, 2013 Accepted: 12 September, 2013

Address for correspondence: Young Ah Lee, MD

Division of Endocrinology and Metabolism, Department of Pediatrics, Seoul National University Children's Hospital, 101 Daehak-ro, Jongno-gu, Seoul 110-744, Korea

Tel: +82-2-2072-2308

Fax: +82-2-743-3455

E-mail:nina337@snu.ac.kr

\section{Introduction}

With the increasing prevalence of type 1 and type 2 diabetes mellitus in children ${ }^{1,2)}$, there is also expected to be an increase in patients transitioning from childhood to adulthood. Preparation for this transition is required on the part of the patient, family members and healthcare providers. According to developmental theorist Arnett ${ }^{3)}$, because of changes in society where the adult role (marriage and parenthood) is delayed until the late twenties, adolescents of today are not expected to immediately take on a role as an adult. Thus a new theory of "emerging adulthood" from the late teens through the twenties (ages, 18-30 years) was proposed. Emerging adulthood can be further classified into early (ages, 18-24 years) and later (ages, 25-30 years) phases. In the early phase, there still exists a conflict between the desire for and a fear of independence from one's parents, however, in the later phase one is able to take on a more traditional adult role. In the case of the diabetic patient, the later phase of emerging adulthood is the critical window of opportunity for health care interventions due to improvements in self-care behavior and better interactions within the medical system. In 2011, the American Diabetes Association (ADA) and a panel of specialists proposed 
recommendations for transition from pediatric to adult diabetes care systems ${ }^{4)}$.

This article explores the critical issues in diabetes care for the emerging adult patient and reviews the 2011 recommendations of the ADA in regards to the transitional care for the diabetic patient diagnosed in childhood.

\section{Issues in the transition between pediatric and adult diabetes care}

For the diabetic patient, the transitional period is characterized by admission to college or a place in the workforce leading to geographic, economic and emotional independence away from the parental home. It is during this period that the patient is exposed to high risk behaviors (smoking, drinking, and drugs) and poor glycemic control. These factors in turn increase the risk for acute diabetic complications, and chronic microvascular and/or macrovascular complications.

\section{Differences between the pediatric and adult diabetes care systems: the need for transitional care}

In the pediatric care system, the patient at the clinic is accompanied by one's parents or guardians and the focus is on total care of the patient within the setting of the family. In contrast, the adult care system focuses on the medical problem at hand and the clinician guides the patient to autonomously make one's own decisions regarding behavior and treatments. A gradual transition from the pediatric to adult care system is recommended.

\section{Poor glycemic control and other risk factors}

There is a difference in the target glycemic control realistically achieved in clinical practice and the glycemic target recommended by the ADA. In a study where type 1 diabetic patients diagnosed in childhood were followed up prospectively for 11 years to ages 17-25 years, glycemic control during the transitional period was consistently poor ${ }^{5)}$. SEARCH for diabetes in Youth study ${ }^{6}$ reported that the percentage of type 1 diabetic patients who achieved recommended levels of glycemic control (glycosylated hemoglobin [HbA1c] $<7.5 \%$ in ages $13-18$ years, HbAlc $<7.0 \%$ in ages $>19$ years) were only $32 \%$ for those $13-18$ years of age and $18 \%$ for those greater than 19 years of age. The Diabetes Control and Complications Trial/Epidemiology of Diabetes Interventions and Complications (DCCT/EDIC) Study ${ }^{7)}$ showed that poor glycemic control during the transitional period is associated with diabetic complications. Furthermore, the risk for cardiovascular complications was increased by poor glycemic control, overweight/obesity and insulin resistance ${ }^{6,8)}$. Thus, cardiovascular complications need to be addressed and managed in the transitional period.

\section{Loss to follow-up}

If the patient fails to show up for clinic appointments, there is greater risk for poor glycemic control. In addition, because of missed screening tests for complications, the risk of development and progression of diabetic complications increases. There are increased visits to the emergency room due to hypoglycemia or hyperglycemia with acute complications like diabetic ketoacidosis as well as the increased risk of chronic complications in the patient with a long time lapse from the pediatric to first adult clinic visit.

\section{Increased risk for acute complications}

There are many reasons for the increased risk of acute complications of hypoglycemia, hyperglycemia, and diabetic ketoacidosis during the transitional period. Contributing factors include decreased parental supervision with frequent loss to follow up appointments, poor diabetic control due to increased pressures at school or work, irregular mealtimes, decreased physical activity and changes in lifestyle including consumption of alcohol. A recent study of continuous glucose monitoring in 15-24 years old diabetic patients showed the prevalence of nighttime hypoglycemia at $8.8 \%{ }^{9)}$. In patients under 30 years of age, acute complications are the main cause of mortality while cardiovascular complications were the main cause of mortality in those past 30 years of age.

\section{Psychosocial issues}

Many stressors are known to act as barriers to effective self-care of the diabetic patient. Stressful encounters with family, friends and coworkers without diabetes, the guilt and anxiety that follows poor self-control, and worries of future development of complications are some of these stressors. There have been previous reports of delayed psychosocial maturity of type 1 diabetic patients compared to healthy controls. However reports since the DCCT study have found no difference between diabetic patients and healthy controls ${ }^{10)}$. Diabetic patients with disordered eating behaviors and those with comorbid psychiatric disorders have been known to be at higher risk for poor glycemic control and development of complications ${ }^{5}$. These patients should be recognized and provided for appropriate care. The prevalence of eating disorders in the female diabetic patient is 2.4 times higher than in the general female population ${ }^{11)}$. Also, there is greater potential for such disorders with $30 \%$ of female patients having skipped insulin injections for weight reduction ${ }^{12)}$. Depression is a well-known risk factor for poor glycemic control and diabetic complications. Depression been reported to be present in $23-35 \%$ of those between the ages of $18-28^{13)}$.

\section{Sexual and reproductive health issues}

All women of reproductive age are at risk of pregnancy and sexually transmitted diseases regardless of the presence of diabetes. In a study of women aged 22-44 years of age, the use of female contraception was lower in the diabetic female when compared to normal controls $(27 \% \text { vs. } 37 \%)^{10)}$. In order to avoid unexpected pregnancies and to better the outcome of pregnancies, careful planning is required. To decrease maternal and fetal risk and give birth to healthy infants, diabetic pregnancies need to be planned with emphasis on strict prenatal glycemic control. 


\section{Alcohol, smoking, and drug abuse}

Consumption of alcohol inhibits gluconeogenesis in the liver which may lead to severe hypoglycemia after drinking. Cigarette smoking is an established risk factor for cardiovascular disease as well as microalbuminuria. Drug abuse increases the risk for acute and chronic diabetes complications. These high risk behaviors also endanger the safety of transitional diabetic patients who start to drive at this age. Thus, drinking and drugs associated with driving must be absolutely prohibited and education regarding prevention of and management of hypoglycemia while driving is required.

\section{Emergence of signs of chronic diabetes complications}

The prevalence of diabetic complications in adolescence is low. However atherosclerotic vascular changes have been reported to develop in early adolescence. Cardiovascular risk is increased with high LDL (low-density lipoprotein) cholesterol, low HDL (high-density lipoprotein) cholesterol, smoking, and high HbAlc levels. Since guidelines for treatment of diabetes complications in adults are based mostly on type 2 diabetes, these guidelines must be individualized for the adult patient with type 1 diabetes. Discussions regarding complications and preparations for the transition of care must take place before the actual transition to adult care systems.

\section{ADA recommendations for care transition of emerging adults with diabetes}

The ADA level-of-evidence grade, as shown in Table 1, is provided in parentheses. This part excerpted ADA position statement (14 recommendations and level of evidence) for emerging adults with diabetes ${ }^{4}$.

1) Pediatric health care providers working collaboratively with the patient and family, should prepare the developing teen for the upcoming transition in health care delivery beginning at least 1 year prior to the transfer to adult health care providers and likely during the early adolescent years (level of evidence: E).

2) Preparation should include a more directed focus on diabetes self-management skills for the teen/emerging adult and his/her parents. Broadening responsibilities go beyond diabetes management tasks such as glucose self-monitoring and insulin

Table 1. American Diabetes Association evidence grading system

\begin{tabular}{|c|c|}
\hline Level of evidence & Description \\
\hline A & $\begin{array}{l}\text { Clear or supportive evidence from well conducted } \\
\text { randomized controlled trials or meta-analyses } \\
\text { that incorporated quality ratings }\end{array}$ \\
\hline B & $\begin{array}{l}\text { Supportive evidence from well-conducted cohort } \\
\text { or observational studies }\end{array}$ \\
\hline C & $\begin{array}{l}\text { Supportive evidence from poorly controlled or } \\
\text { uncontrolled studies, or conflicting evidence } \\
\text { with the weight of evidence supporting the } \\
\text { recommendation }\end{array}$ \\
\hline E & Expert consensus or clinical experience \\
\hline
\end{tabular}

Adapted from Peters et al. Diabetes Care 2011;34:2477-854). administration and should include scheduling appointments and ensuring a proper supply of medications and supplies. Diabetes education should be redirected to the growing and developing teen rather than focused on the parents (level of evidence: E).

3) Preparation should include information about the differences between pediatric and adult providers in their approaches to care, as well as education regarding health insurance options and how to maintain coverage (level of evidence: E).

4) The pediatric provider should prepare and provide to both the patient and future adult care provider a written summary that includes an active problem list, compilation of medications, assessment of diabetes self-care skills, summary of past glycemic control and diabetes related comorbidities, as well as a summary of any mental health problems and referrals during pediatric care (level of evidence: E).

5) Health care providers need to recognize the vulnerability of emerging adults with diabetes to loss of consistent health care and difficulties in adhering to diabetes management due to competing psychosocial, educational, and vocational changes, leading to deteriorating glycemic control. Both pediatric and adult care providers should assist in providing support and links to resources that could benefit the patient (level of evidence: B).

6) The transferring health care providers should provide emerging adults with specific referrals to adult care providers versed in the principles of intensive diabetes management to match the particular needs of the patients with type 1 or type 2 diabetes. One might consider creation of a directory of adult providers with expertise and interest in the care of young adults (level of evidence: E)

7) Consideration should be given to assisting the young adult with scheduling the first appointment with the adult care provider within 3-4 months of the final pediatric visit (level of evidence: $\mathrm{C}$ ).

8) Care must be individualized and developmentally appropriate, with an emphasis on adherence to diabetes self-management and consistent use of glucose-lowering medications in order to prevent acute and long-term complications of diabetes (level of evidence: B).

9) Emerging adults with diabetes should be evaluated and treated for disordered eating behaviors and affective disorders. It is important for the diabetes provider to have a mental health referral source who understands the fundamentals of working with individuals with diabetes (level of evidence: C).

10) Ongoing visits should occur every 3 months for patients taking insulin and every 3-6 months for patients with type 2 diabetes not taking insulin, according to current ADA recommendations (level of evidence: $\mathrm{E}$ ).

11) Screening guidelines for microvascular and macrovascular complications in pediatric and adult patients with diabetes should be followed (level of evidence: B).

12) Assessment of risk for macrovascular complications should begin in childhood according to guidelines for lipid screening, blood pressure assessment, and weight management. Management of lipids and hypertension should proceed 
according to pediatric and adult guidelines (level of evidence: B).

13) Birth control, pregnancy planning and risks, prevention of sexually transmitted illnesses, use of alcohol and drugs, smoking, and driving should be discussed with the older teens and the emerging adults by both pediatric and adult providers with an emphasis on the interplay of these issues with diabetes (level of evidence: E).

14) Both pediatric and adult providers should ensure that their patients with diabetes receive ongoing primary and preventive health care (usually separate from their ongoing diabetes specialty care) and, ideally, that the emerging adult feels that he/she is receiving accessible, patient-centered, coordinated, comprehensive, continuous, compassionate, and culturally effective care (level of evidence: E).

These 2011 recommendations by the $\mathrm{ADA}^{4)}$ are limited by the lack of data regarding posttransition outcomes and best practices in transition care ${ }^{14)}$. Transitions in Care: Meeting the Challenges of Type 1 Diabetes in Young Adults ${ }^{15)}$, published by the ADA provides information for the patient "getting prepared for your journey with diabetes through young adult," the parents "helping your child during the transition to young adult" and the clinician "clinical principles for the health care professional." A brief summary of the recommendations for the health care provider of the transitional diabetic patient follows.

1) Open communication between the patient and health care provider is most important in transitional care.

2) The health care provider should act as a coach to help the patient in self-management and self-care.

-Advice for patien's journey ${ }^{15)}$

(1) Keep in mind that it will take a while for all your diabetes self-care tasks to become an automatic part of your daily routine. Achieving good diabetes control inevitably demands extra effort. Unfortunately it's easy to get burnt out by the new demands and then, before long, decide that the effort isn't worthwhile. Don't let this happen to you.

(2) Don't view lapses as a sign of failure

(3) Set attainable goals

(4) Everyone gets on track at a different pace

3) Barriers to self-management and care must be recognized and solutions sought. Fears of hypoglycemia, worries of weight gain, diabetes burnout due to repeated failures in attaining unrealistic glucose targets, eating disorders and other psychiatric problems were possible barriers.

4) Attainable goals and a realistic treatment plan should be set in collaboration with the patient. By being able to achieve realistic goals, the patient does not become discourage and gains confidence in self-care.

5) Expressions of pressure to attain glycemic goals or anticipatory behavior should be avoided. When referring to glycemic control, expressions such as "bad" or "good" should be avoided. Neutral expressions such as "beyond normal range" or "high" are recommended.

6) Increasing the patient's fears and anxieties of diabetic complications should be avoided. Fear-mongering of possible complications does not motivate better glycemic control.
7) Issues of the transitional period including alcohol, smoking, drinking, sexual activity, pregnancy and sick days should be discussed.

8) If the relationship between the patient and the pediatric health care provider is solid, the transition to an adult health care provider can be delayed until the patient is ready for selfcare and can be expected to take on an adult role.

The goals of transitional care are to effectively transition the diabetic patient from the pediatric to adult care system with less elapsed time in between and to improve posttransition outcome. Passage to adult medical care has been shown to improve with transition to an adult health care provider after a period of concurrent pediatric and adult medical care or to transition to an adult health care provider within the same center, rather than referring the patient to another center ${ }^{16)}$. The presence of a navigator coordinator who provided telephone and email contact with young adults to provide support in accessing transitional adult medical care had the effect of lowering rates of loss to follow up and short term medical outcomes ${ }^{17)}$. A systemic review of supporting programs of diabetes transitional care ${ }^{18-21)}$ showed that patient education programs, medical coordinators and auxiliary service systems were beneficial in improving glycemic control and decreasing complications and loss to follow up ${ }^{22)}$. Although some reports show no changes in posttransition $\mathrm{HbAlc} \mathrm{c}^{16,23)}$ or even an increase $\mathrm{e}^{24)}$ in $\mathrm{HbAlc}$, others have reported improvements with transition coordination intervention programs ${ }^{18,19)}$. Further studies are needed in regards to those practices in transition care that can bring about improvements in post-transition outcome.

\section{Conclusions}

Providing appropriate transitional care for the emerging adult is imperative in preventing complications and maintaining health throughout adult life. Programs of diabetes transition care $^{18-21)}$ have reported favorable results. Further studies are needed to provide evidence based transitional care programs that take both medical and psychosocial aspects of diabetes care into consideration.

\section{Conflict of interest}

No potential conflict of interest relevant to this article was reported.

\section{References}

1. Patterson CC, Dahlquist GG, Gyurus E, Green A, Soltesz G; EURODIAB Study Group. Incidence trends for childhood type 1 diabetes in Europe during 1989-2003 and predicted new cases 2005-20: a multicentre prospective registration study. Lancet 2009;373:2027-33.

2. Pinhas-Hamiel O, Zeitler P. The global spread of type 2 diabetes mellitus in children and adolescents. J Pediatr 
2005; 146:693-700.

3. Arnett JJ. Emerging adulthood: a theory of development from the late teens through the twenties. Am Psychol 2000;55:469-80.

4. Peters A, Laffel L; American Diabetes Association Transitions Working Group. Diabetes care for emerging adults: recommendations for transition from pediatric to adult diabetes care systems: a position statement of the American Diabetes Association, with representation by the American College of Osteopathic Family Physicians, the American Academy of Pediatrics, the American Association of Clinical Endocrinologists, the American Osteopathic Association, the Centers for Disease Control and Prevention, Children with Diabetes, The Endocrine Society, the International Society for Pediatric and Adolescent Diabetes, Juvenile Diabetes Research Foundation International, the National Diabetes Education Program, and the Pediatric Endocrine Society (formerly Lawson Wilkins Pediatric Endocrine Society). Diabetes Care 2011;34:2477-85.

5. Bryden KS, Dunger DB, Mayou RA, Peveler RC, Neil HA. Poor prognosis of young adults with type 1 diabetes: a longitudinal study. Diabetes Care 2003;26:1052-7.

6. Petitti DB, Klingensmith GJ, Bell RA, Andrews JS, Dabelea D, Imperatore G, et al. Glycemic control in youth with diabetes: the SEARCH for diabetes in Youth Study. J Pediatr 2009;155:668-72.e1-3.

7. Diabetes Control and Complications Trial/Epidemiology of Diabetes Interventions and Complications (DCCT/ EDIC) Research Group, Nathan DM, Zinman B, Cleary PA, Backlund JY, Genuth S, et al. Modern-day clinical course of type 1 diabetes mellitus after 30 years' duration: the diabetes control and complications trial/epidemiology of diabetes interventions and complications and Pittsburgh epidemiology of diabetes complications experience (19832005). Arch Intern Med 2009;169:1307-16.

8. Copeland KC, Zeitler P, Geffner M, Guandalini C, Higgins J, Hirst K, et al. Characteristics of adolescents and youth with recent-onset type 2 diabetes: the TODAY cohort at baseline. J Clin Endocrinol Metab 2011;96:159-67.

9. Juvenile Diabetes Research Foundation Continuous Glucose Monitoring Study Group. Prolonged nocturnal hypoglycemia is common during 12 months of continuous glucose monitoring in children and adults with type 1 diabetes. Diabetes Care 2010;33:1004-8.

10. Weissberg-Benchell J, Wolpert H, Anderson BJ. Transitioning from pediatric to adult care: a new approach to the post-adolescent young person with type 1 diabetes. Diabetes Care 2007;30:2441-6.
11. Jones JM, Lawson ML, Daneman D, Olmsted MP, Rodin G. Eating disorders in adolescent females with and without type 1 diabetes: cross sectional study. BMJ 2000;320:1563-6.

12. Polonsky WH, Anderson BJ, Lohrer PA, Aponte JE, Jacobson AM, Cole CF. Insulin omission in women with IDDM. Diabetes Care 1994;17:1178-85.

13. Hislop AL, Fegan PG, Schlaeppi MJ, Duck M, Yeap BB. Prevalence and associations of psychological distress in young adults with Type 1 diabetes. Diabet Med. 2008;25:91-6.

14. Garvey KC, Wolpert HA, Rhodes ET, Laffel LM, Kleinman $\mathrm{K}$, Beste MG, et al. Health care transition in patients with type 1 diabetes: young adult experiences and relationship to glycemic control. Diabetes Care 2012;35:1716-22.

15. Wolpert AB, Anderson BJ, Weissberg-Benchell J. Transitions in care: meeting the challenges of type 1 diabetes in young adults. Virginia: American Diabetes Association, 2009.

16. Sparud-Lundin C, Ohrn I, Danielson E, Forsander G. Glycaemic control and diabetes care utilization in young adults with Type 1 diabetes. Diabet Med 2008;25:968-73.

17. Van Walleghem N, Macdonald CA, Dean HJ. Evaluation of a systems navigator model for transition from pediatric to adult care for young adults with type 1 diabetes. Diabetes Care 2008;31:1529-30.

18. Cadario F, Prodam F, Bellone S, Trada M, Binotti M, Trada M, et al. Transition process of patients with type 1 diabetes (T1DM) from paediatric to the adult health care service: a hospital-based approach. Clin Endocrinol (Oxf) 2009;71:346-50.

19. Holmes-Walker DJ, Llewellyn AC, Farrell K. A transition care programme which improves diabetes control and reduces hospital admission rates in young adults with Type 1 diabetes aged 15-25 years. Diabet Med 2007;24:764-9.

20. Lane JT, Ferguson A, Hall J, McElligott M, Miller M, Lane $\mathrm{PH}$, et al. Glycemic control over 3 years in a young adult clinic for patients with type 1 diabetes. Diabetes Res Clin Pract 2007;78:385-91.

21. Nakhla M, Daneman D, To T, Paradis G, Guttmann A. Transition to adult care for youths with diabetes mellitus: findings from a Universal Health Care System. Pediatrics 2009; 124:e1134-41.

22. Crowley R, Wolfe I, Lock K, McKee M. Improving the transition between paediatric and adult healthcare: a systematic review. Arch Dis Child 2011;96:548-53.

23. Kipps S, Bahu T, Ong K, Ackland FM, Brown RS, Fox CT, et al. Current methods of transfer of young people with Type 1 diabetes to adult services. Diabet Med 2002;19:649-54.

24. Lotstein DS, Seid M, Klingensmith G, Case D, Lawrence JM, Pihoker C, et al. Transition from pediatric to adult care for youth diagnosed with type 1 diabetes in adolescence. Pediatrics 2013;131:e1062-70. 